\title{
A review of screening strategies for cervical cancer in human immunodeficiency virus-positive women in sub-Saharan Africa
}

This article was published in the following Dove Press journal:

International Journal of Women's Health

2 February 2017

Number of times this article has been viewed

\author{
Manuela Viviano' \\ Pierre DeBeaudrap ${ }^{2}$ \\ Pierre-Marie Tebeu ${ }^{3}$ \\ Jovanny T Fouogue ${ }^{3}$ \\ Pierre Vassilakos ${ }^{4}$ \\ Patrick Petignat' \\ 'Gynecology Division, Geneva \\ University Hospitals, Geneva, \\ Switzerland; ${ }^{2}$ Centre Population et \\ Développement - UMR 196, Institute \\ of Research for the Development, \\ University of Paris-Descartes, Paris, \\ France; ${ }^{3}$ Faculty of Medicine and \\ Biomedical Sciences and Centre \\ Hospitalier Universitaire (CHUY), \\ Yaoundé, Cameroon, Africa; ${ }^{4}$ Geneva \\ Foundation for Medical Education and \\ Research, Geneva, Switzerland
}

Correspondence: Manuela Viviano Gynecology Division, Geneva University Hospitals, Boulevard de la Cluse 30, I205 Geneva, Switzerland Email manuela.viviano@gmail.com

\begin{abstract}
Cervical cancer (CC) is a leading cause of cancer-related death and a major public health issue in sub-Saharan Africa. This heavy burden parallels that of the human immunodeficiency virus (HIV) infection, which increases the risk of developing CC. Despite the progressive reduction of HIV prevalence in the past decade, the CC incidence and mortality rates in sub-Saharan Africa remain high. The heterogeneity of the distribution of the two diseases in the African continent, together with the different availability of human and material resources, stands in the way of finding an appropriate screening strategy. The lack of high-quality evidence on the prevention of CC for HIV-positive women, which is necessary for the implementation of efficient screening and treatment strategies, results in the absence of a clearly defined program, which is responsible for the low screening uptake and high mortality rates in sub-Saharan Africa. By taking advantage of the HIV-positive women's frequent access to health facilities, one way to increase the $\mathrm{CC}$ screening coverage rates would be by providing integrated HIV and screening services within the same infrastructure. With the increasing availability of cost-effective methods, screening is becoming more and more available to women who have limited access to health care. Moreover, the introduction of point-of-care technologies for human papillomavirus testing and the subsequent implementation of screen-and-treat strategies, by reducing the number of clinical appointments and, in the long term, the loss to follow-up rates, open up new opportunities for all women, regardless of their HIV status. The purpose of this review is to provide an insight into the different screening practices for $\mathrm{CC}$ in order to help define one that is adapted to the resources and necessities of HIV-positive women living in middle-to-low income countries.
\end{abstract}

Keywords: cervical cancer, screening, human papillomavirus (HPV), human immunodeficiency virus (HIV)

\section{Introduction}

As the prevalence of the human immunodeficiency virus (HIV) infection and that of cervical premalignant lesions in certain regions reach $20 \%$ and $9 \%$, respectively, these two diseases represent two important public health issues in sub-Saharan Africa. ${ }^{1-4}$ Since the onset of the HIV epidemic in the late 1970s, the US Center for Disease Control and Prevention (CDC) has labeled cervical cancer (CC) as an acquired immunodeficiency syndrome (AIDS)-defining disease. ${ }^{5}$ The global incidence of CC in sub-Saharan Africa ranges from 43.3/100,000 to 69.8/100,000 women, as opposed to an incidence of 9/100,000 to 12.8/100,000 reported in Europe. ${ }^{6-9}$

A growing body of evidence supports the fact that human papillomavirus (HPV) infection is the main cause for the development of cervical intraepithelial neoplasia (CIN) and invasive CC..$^{10}$ Infection with HIV significantly increases a woman's risk 
of developing cervical premalignant lesions and cancer by impairing her natural cell-mediated immune response, and thereby, altering her capacity of properly eliminating HPV. ${ }^{11-13}$ Furthermore, evidence coming from studies conducted both in industrialized and developing countries has shown that the risk of developing cervical premalignant lesions increases as the CD4 count lowers. ${ }^{14,15}$ This association could be more relevant in sub-Saharan Africa, where access to highly active antiretroviral therapy (HAART) and subsequent retention in care are still real challenges. ${ }^{16,17}$

The development of health care infrastructure, together with an increased access to HAART, has significantly decreased the worldwide AIDS-related mortality over the past few years. ${ }^{18}$ In sub-Saharan Africa, in particular, the World Health Organization's (WHO) 2015 guidelines have extended the treatment criteria initiation to all patients, regardless of their CD4+ count, in an effort to make more HIV-positive people eligible for therapy. ${ }^{19}$ This translates into an increased life expectancy for HIV-infected women, which subsequently exposes them to the risk of developing other AIDS-defining cancers. Whereas most AIDS-related diseases are progressively becoming less frequent, CC's incidence has not substantially decreased with the growing use of antiretroviral therapy. ${ }^{20}$

In the 2014 guidelines, the WHO has stated the need to fulfill the research gap in the CC screening and follow-up of HIV-positive women living in developing countries. ${ }^{21}$ As the lack of good quality evidence makes it difficult to define the screening intervals and modalities for HIV-infected patients, the current guidelines for this high-risk group of women are mostly based on expert opinion. ${ }^{21}$

The aim of the present study is to provide an overview of the current CC screening strategies for HIV-infected women and to discuss the current issues and research gap on the subject to the extent of defining the best possible screening approach for a disease that currently has a large impact on the women's quality of life and expectancy.

\section{Overview of the current screening strategies and issues}

The incidence rates of $\mathrm{CC}$ in Africa vary widely by geographical region. ${ }^{6}$ Figures 1 and 2 show the distribution of the HIV epidemic and the incidence of $\mathrm{CC}$ in the different African countries. Despite the growing evidence supporting the association between HIV and HPV infection, the mechanisms resulting in CC are not fully understood, especially for HIV-positive women. ${ }^{22-25}$ This finding has been confirmed by a study conducted in South Africa and Burkina Faso, which found similar HIV infection rates in the two settings but significantly higher rates of cervical precancerous lesions in South Africa. ${ }^{26}$ The causes of this heterogeneity remain unclear and may partly be attributed to genetics, lifestyles, environmental factors, and migration patterns. These unregulated trends may explain why the

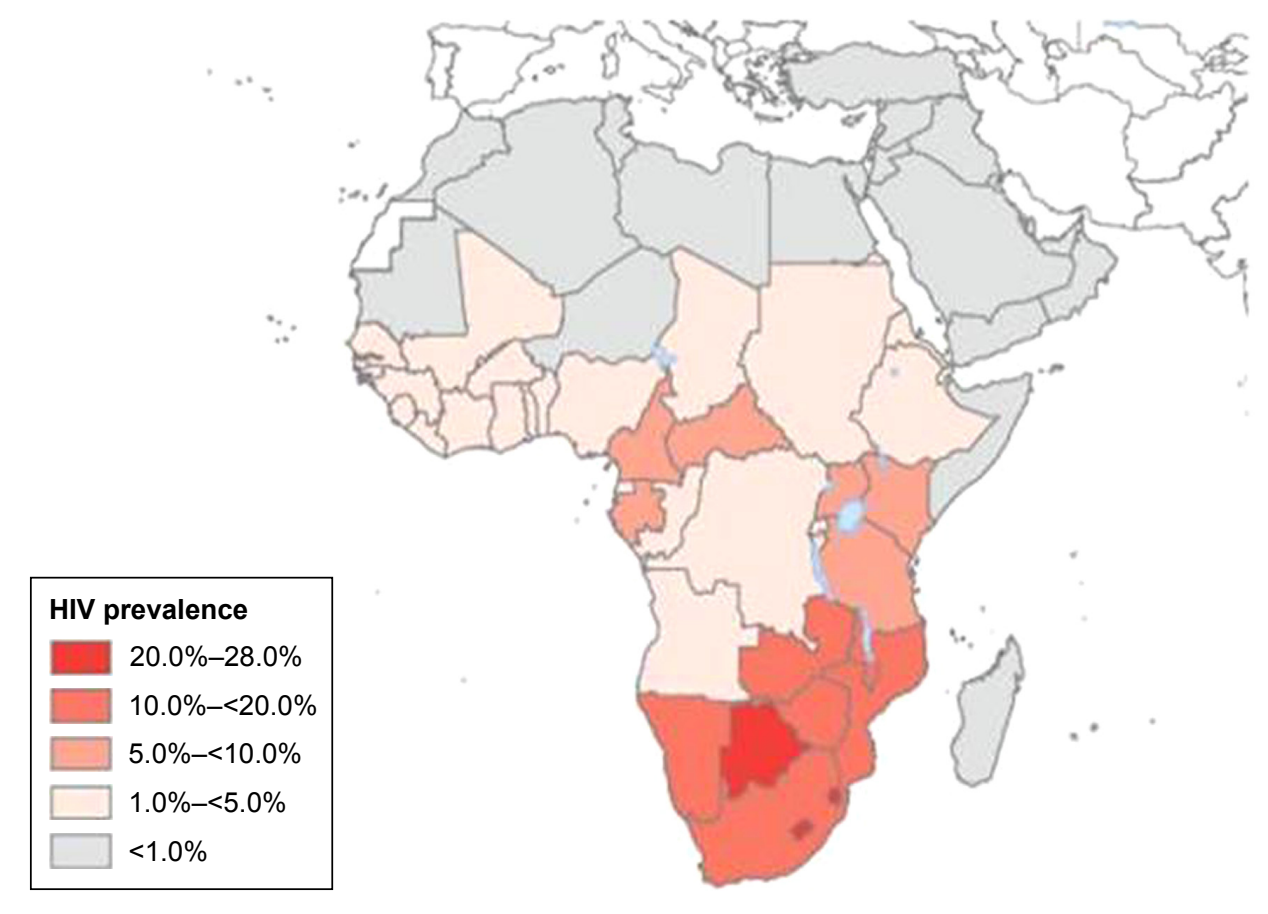

Figure I Global distribution of HIV in Africa in 2007.

Note: Reproduced from Hotez PJ, Fenwick A, Kjetland EF. Africa's 32 cents solution for HIV-AIDS. PLoS Negl Trop Dis. 2009;3(5):e430. ${ }^{86}$ 


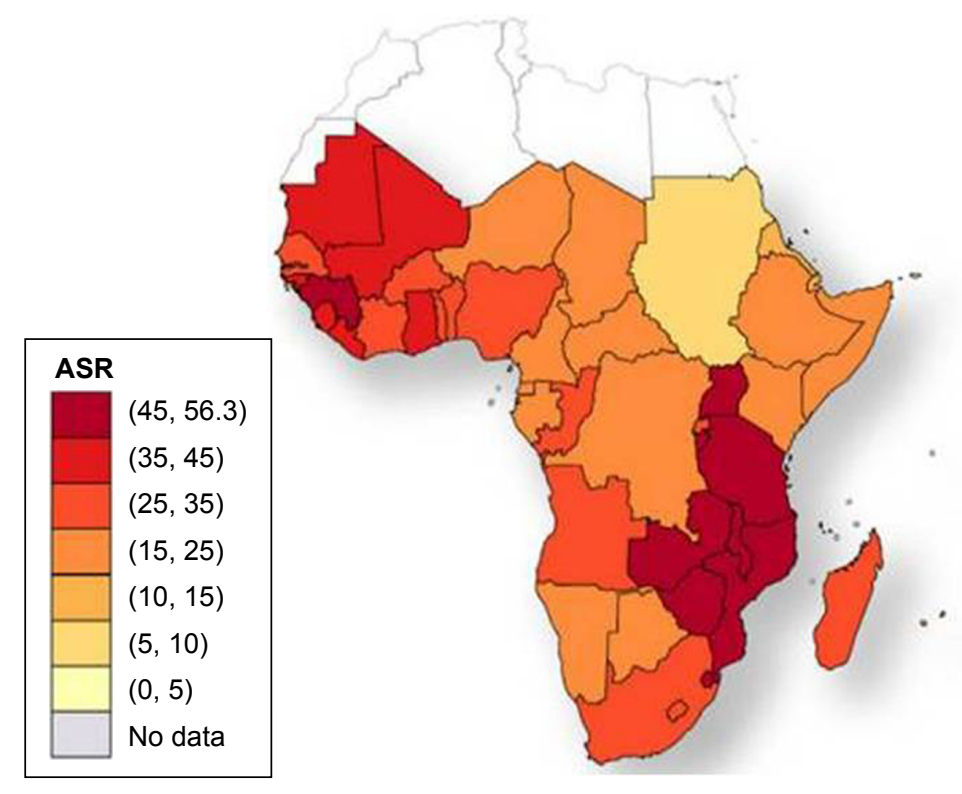

Figure 2 Cervical cancer age-standardized incidence rates per 100,000 women in 2008.

Note: Reproduced with permission from Ferlay J, Shin HR, Bray F, Forman D, Mathers C and Parkin DM. GLOBOCAN 2008, Cancer Incidence and Mortality Worldwide: IARC CancerBase No. 10 [Internet]. Lyon, France: International Agency for Research on Cancer; 2010.87

Abbreviation: ASR, age-standardized incidence rates per 100,000 women and year.

reported CC screening tests' positivity rates vary widely by country. ${ }^{27,28}$ The geographical variations in the screening tests' performance are partly responsible for the difficulty in establishing an efficient screening strategy that is adapted to the resources and capacity of each region.

Despite the accuracy of Pap testing in the detection of cervical lesions among HIV-positive women, the high costs, and the need of human and material resources imply the necessity to consider other alternatives for CC screening in Africa. ${ }^{29}$ One significant limitation of the current cytology-based screening strategy for the identification of cervical precancerous lesions is represented by the high rate of false negative test results, which have been found to be more frequent in HIV-positive women when compared to the general population. ${ }^{30}$ Based on this finding, it has been suggested that colposcopy be performed routinely to screen HIV-positive women, which would increase the necessity of paramount human, financial, and material resources. ${ }^{31}$ In addition, the use of Pap test-based screening in low- and middle-income countries (LMIC) has proven to be difficult to provide due to the limited resources for the pathological diagnosis and the significant delay between the primary screening visit and the subsequent treatment, leading to loss to follow-up, and in the long term, disease progression. ${ }^{32}$

In order to overcome the main obstacles to screening uptake, a reevaluation of the traditional cytology-based screening strategy is advised, with the ultimate aim of extending screening coverage to those women who are, in relation to their HIV status, exposed to a higher risk of developing invasive $\mathrm{CC}$.

\section{Visual inspection methods}

The visual inspection with acetic acid (VIA) approach for screening prior to treatment of cervical precancerous lesions in the context of a "screen-and-treat" protocol in LMIC is currently recommended by the WHO guidelines. ${ }^{21}$ This method is often coupled with visual inspection with Lugol's iodine, which has demonstrated to improve the performance of VIA on HIV-positive women. ${ }^{33}$

As shown in the decision-making algorithm in Figure 3, one of the main strengths of visual inspection methods is the prompt availability of the test results, which allows the immediate treatment of screen-positive women. This particular aspect allows health services to contrast the high dropout rates associated with the multiple-visit, cytology-based screening approach, which have proven to reach as much as $80 \%$ of women screened in sub-Saharan Africa. ${ }^{34}$ Several studies have demonstrated that, while being safe and cost-effective, the VIA-based screening approach results in a significant reduction of loss to follow-up among HIV-infected women within a low-resource setting. ${ }^{35-38}$ Moreover, the overall acceptance rates of HIV-positive women who were offered this screening technique varied from $86 \%$ in a study conducted in Mozambique to as much as $96.5 \%$ in a study conducted in Nigeria, thus indicating that the majority of women will accept screening for $\mathrm{CC}$ 


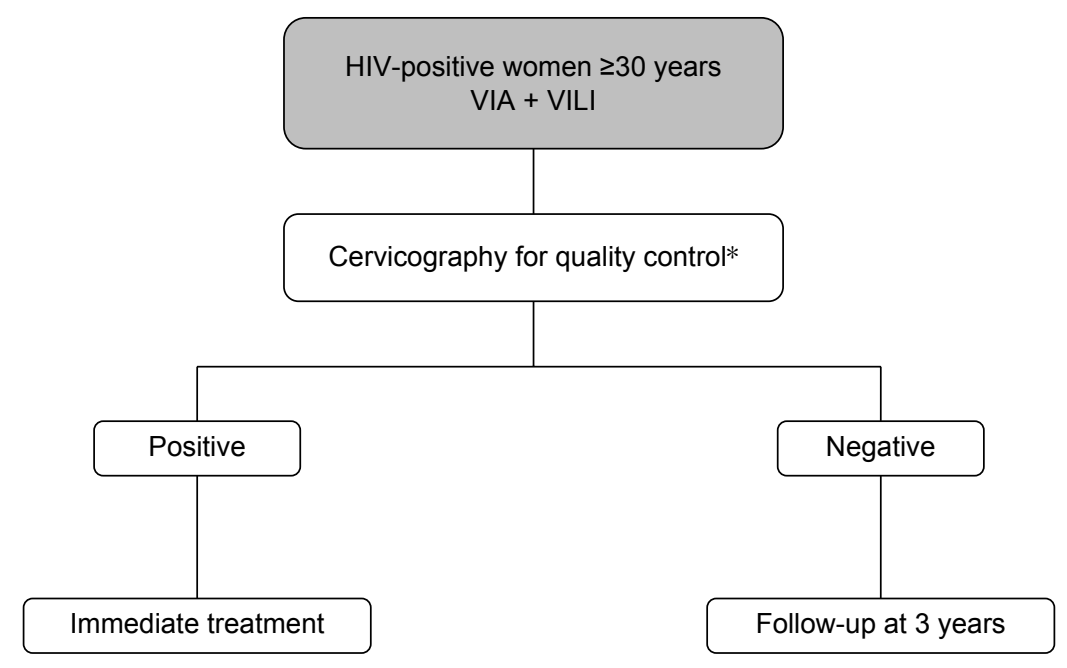

Figure 3 Decision-making algorithm for VIA-based screening.

Note: *If available.

Abbreviations: VIA, visual inspection with acetic acid; VILI, visual inspection with Lugol's iodine.

with VIA if proactively offered the opportunity to test. ${ }^{39,40}$ In addition, a multicenter study conducted in sub-Saharan Africa has found that the majority of HIV-infected VIApositive women were eligible to promptly undergo treatment with cryotherapy, thus further supporting the efficacy of this primary screening method. ${ }^{35}$ The VIA-based "screen-andtreat" strategy embodied in an HIV service platform has been implemented in Zambia in 2006 and has ever since provided CC screening service to over 100,000 women. Although the program initially targeted only HIV-infected patients, its use has been rapidly extended to all women, regardless of their HIV status, with the aim of improving health education and reducing HIV stigmatization. ${ }^{41}$

As a screening test to detect CIN grade 2 or worse (CIN2+) among HIV-infected women, VIA has shown a good performance in a large body of studies conducted in sub-Saharan Africa. ${ }^{33,37}$ The different specificity and sensitivity values of VIA across different studies conducted in sub-Saharan Africa are reported in Table 1. As the lesions occupy a larger area of the cervix, the VIA positivity rates have proven to be higher among HIV-positive women when compared to their HIV-negative counterparts. ${ }^{35}$ The main drawback of this technique is the subjectivity of the diagnosis, which is influenced by the health worker's qualitative judgment and experience, and the environmental conditions, in which the examination takes place. In parallel, to what is observed among HIV-negative women, these aspects result in high false positive rates, which limit the technique's specificity and lead to a risk of overtreatment. ${ }^{42,43}$ The subjectivity of the diagnosis obtained with visual inspection methods can be minimized by the use of digital cervicography. The use of digital images of the cervix allows users to standardize the visual inspection examination while providing a quality control system. ${ }^{44}$ Although this technique may seem inappropriate for low-resource settings due to the additional economic, material, and human requirements for its use, several studies have shown its successful implementation in different sub-Saharan African regions. ${ }^{45,46}$

While the VIA result interpretation, depending mainly on the examiner's judgment, leads mainly to false positive

Table I Sensitivity and specificity values of VIA among HIV-positive women across different studies

\begin{tabular}{|c|c|c|c|c|c|c|}
\hline References & Country & $\mathbf{N}$ & HIV status & Reference test & Sensitivity (\%) & Specificity (\%) \\
\hline Akinwuntwan et al ${ }^{88}$ & Nigeria & 205 & HIV-positive & Histology (CIN2+) & 76 & 83 \\
\hline Mabeya et a ${ }^{89}$ & Kenya & 150 & HIV-positive & Histology (CIN2+) & 70 & 51 \\
\hline Kuhn et $\mathrm{a}^{90}$ & South Africa & 956 & Mixed & Histology (CIN2+) & 64 & 74 \\
\hline Fihrnaber et $\mathrm{a}^{91}$ & South Africa & 1,202 & HIV-positive & Histology (CIN2+) & 65 & 69 \\
\hline Dartell et al ${ }^{92}$ & Tanzania & 3,603 & Mixed & Cytology (HSIL+) & 50 & 91 \\
\hline Chung et $\mathrm{al}^{29}$ & Kenya & 500 & HIV-positive & Histology (CIN2+) & 63 & 66 \\
\hline Chibwesha et $\mathrm{al}^{50}$ & Zambia & 200 & HIV-positive & Histology (CIN2+) & 48 & 92 \\
\hline
\end{tabular}

Abbreviations: $\mathrm{CIN} 2+$, cervical intraepithelial neoplasia grade 2 or worse; HIV, human immunodeficiency virus; HSIL+, high-grade squamous intraepithelial lesion; VIA, visual inspection with acetic acid. 
diagnoses, it may also be responsible for a certain number of false negatives. A recent cost-effectiveness analysis comparing different $\mathrm{CC}$ screening methods has demonstrated that, although VIA is the most cost-effective option when compared to cytology and HPV testing, its lower sensitivity when compared to other screening techniques translates into a significantly increased number of missed diagnoses. ${ }^{47}$ The high rates of false negative diagnoses represent one of the main challenges to the use of this technique for primary screening, as they impose the need of multiple-day, longdistance visits, which are responsible for loss to follow-up. As same day treatment with procedures such as cryotherapy or thermocoagulation has proven to be feasible and wellaccepted by patients, one way to reduce loss to follow-up would be to treat all women whose diagnosis is suspicious for a cervical premalignant lesion, although at the cost of increasing the risk of overtreatment. ${ }^{37,48}$

Despite the technological progress that allows the continuous optimization of this screening method, several studies have found that, in addition to user-dependent factors, there are also patient-related factors, such as age and the presence of vaginal infections, which can influence the capacity to read the VIA results on HIV-positive patients, thus leading to possible test result misinterpretation. ${ }^{49}$

The limited reliability of visual inspection methods, together with the reluctance expressed by some women to undergo a screening service that involves a pelvic examination, have given way to the introduction of alternative methods, all while taking into account the main human and institutional barriers that keep the current screening coverage rates in sub-Saharan Africa low.

\section{HPV testing}

HPV testing has proven to be significantly more sensitive in identifying cervical precancerous lesions when compared with cytology, although at the cost of a lower specificity. ${ }^{29,50}$ The different sensitivity and specificity values of HPV testing obtained from studies conducted in sub-Saharan Africa are reported in Table 2. A randomized trial conducted in South Africa has shown that, among 100 screened women, the HPV-based screen-and-treat approach can prevent 12 cases of CIN2+ among HIV-infected women and 3 cases of CIN2+ among HIV-negative patients, whereas VIA can prevent only 7 and 1 case of CIN2+ among HIV-infected and HIVuninfected women, respectively. ${ }^{51}$

The availability of point-of-care technologies for the detection of HPV, which require limited infrastructure and provide test results within 1 hour, potentially allow the implementation of a same day screen-and-treat approach. ${ }^{52}$ One of these is GeneXpert (GeneXpert ${ }^{\mathbb{B}}$; Cepheid, Sunnyvale, CA, USA) which, when used for HPV sample analysis, has shown a higher positive predictive value and sensitivity in the detection of CIN2+ among HIV-positive women when compared to their HIV-negative counterparts. ${ }^{50}$ Similar results have been observed when using the Hybrid Capture 2 DNA assay (Qiagen, Germantown, MD, USA). ${ }^{53}$

Due to the high prevalence of HPV infection among HIV-infected women, the HPV test's specificity remains limited. ${ }^{52,54}$ The results from a study conducted in Kenya suggest that decreased HPV specificity is independently associated with age $<40$ years. ${ }^{29}$ This finding is consistent with the progressive decrease of HPV infection starting at the age 30 years, whereas the peak incidence of CIN occurs about 10 years later. ${ }^{55,56}$ For this reason, similar age restrictions to those applied in the USA for HPV screening, which is currently limited to women aged 30 years and older, should apply to HIV-positive women. ${ }^{57}$

One way to increase the HPV test's specificity and to potentiate its effectiveness is by combining HPV testing with triage methods. One study conducted in Kenya has

Table 2 Sensitivity and specificity of HPV testing among HIV-positive women across different studies

\begin{tabular}{|c|c|c|c|c|c|c|c|}
\hline References & Country & $\mathbf{N}$ & HIV status & Test used & Reference test & Sensitivity (\%) & Specificity (\%) \\
\hline Fihrnaber et $a^{91}$ & South Africa & 1,202 & HIV-positive & HC2 (Qiagen) & Histology (CIN2+) & 92 & 51 \\
\hline Kuhn et a $\left.\right|^{90}$ & South Africa & 956 & Mixed & HC2 (Qiagen) & Histology (CIN2+) & 94 & 64.4 \\
\hline Chibwesha et $\mathrm{a}^{50}$ & Zambia & 200 & HIV-positive & GeneXpert & Histology (CIN2+) & 88 & 60 \\
\hline Dartell et al ${ }^{92}$ & Tanzania & 3,603 & Mixed & HC2 (Digene) & Cytology (HSIL+) & 94.2 & 82.8 \\
\hline Chung et $\mathrm{a}^{29}$ & Kenya & 500 & HIV-positive & Enzyme immunoassay & Histology (CIN2+) & 83.6 & 55.7 \\
\hline Adamson et a ${ }^{65}$ & South Africa & 308 & HIV-positive & $\begin{array}{l}\text { Aptima HPV mRNA assay } \\
\text { on self-collected samples }\end{array}$ & $\begin{array}{l}\text { HPV mRNA assay on } \\
\text { clinician-collected samples }\end{array}$ & 77.4 & 77.7 \\
\hline Segondy et $\mathrm{al}^{26}$ & South Africa & 943 & HIV-positive & careHPV and INNO-LiPA & Histology (CIN2+) & $\begin{array}{l}93.3 \text { (careHPV) } \\
96.7 \text { (INNO-LiPA) }\end{array}$ & $\begin{array}{l}57.9 \text { (careHPV) } \\
32.0 \text { (INNO-LiPA) }\end{array}$ \\
\hline Ngou et a $\left.\right|^{93}$ & $\begin{array}{l}\text { South Africa; } \\
\text { Burkina Faso }\end{array}$ & 160 & HIV-positive & $\mathrm{HC2}$ and INNO-LiPA & Histology (CIN2+) & $\begin{array}{l}88.8 \text { (HC2) } \\
92.5 \text { (INNO-LiPA) }\end{array}$ & $\begin{array}{l}55.2 \\
32.1\end{array}$ \\
\hline
\end{tabular}

Abbreviations: CIN2+, cervical intraepithelial neoplasia grade 2 or worse; HC2, Hybrid Capture 2; HIV, human immunodeficiency virus; HPV, human papillomavirus; HSIL+, high-grade squamous intraepithelial lesion. 
demonstrated that the already high sensitivity of HPV testing in HIV-positive women can be further increased by pairing it up with a triage test, such as VIA or cytology. ${ }^{29}$ The prevalence of specific high-risk HPV types such as HPV-52, HPV-58, HPV-35, and HPV-16 in HIV-positive women is itself a predictor for cervical disease that can be assessed through genotyping. ${ }^{58}$ Other studies have evaluated the use of alternative triage methods for HPV-positive women. One of these is the identification of E6 protein because real-time nucleic acid sequence-based amplification has proven to allow type-specific detection of E6 mRNA from high-risk HPV types. ${ }^{50,59}$ Another triage method is the use of methylation markers of host cell genes involved in cervical carcinogenesis, such as CADM1, MAL, and MIR124-2 loci. It has been demonstrated that the combined use of these methylation markers for HIV-infected HPV-positive women is comparable to that of cytology and superior to HPV16/18 genotyping and VIA for the detection of CIN. ${ }^{60}$

Another study has found that the molecular expression of the insulin-like growth factor II mRNA binding protein 3 (IMP3) and of p16 are significantly higher among HIVinfected women with squamous intraepithelial neoplasia (SIL). ${ }^{61}$ Moreover, the expression of IMP3 among HIVpositive women is more sensitive than p16 in identifying patients at risk for progression and recurrence of high-grade SIL (HSIL).${ }^{61}$ Although this strategy was evaluated solely on clinician-collected cervical samples, it represents another step toward the adoption of molecular tests as a complementary tool for CC screening.

The advantage of HPV genotyping, OncoE6, methylation, and other molecular markers is that they can be assessed in the same cervical sample that is used for primary screening, thus avoiding the need of multiple visits. This aspect makes the molecular screening strategy particularly promising in LMIC, where it could circumvent some of the main practical barriers to $\mathrm{CC}$ screening attendance, such as lack of time and unwillingness to undergo multiple clinical visits that entail one or more pelvic examinations. ${ }^{60,62}$ The decision-making algorithm for HPV-based screening is shown in Figure 4.

Another advantage to HPV testing is the possibility of performing self-sampling. The availability of self-screening represents an additional strategy to improve screening coverage in LMIC, where the limited infrastructure and health personnel reduce the effectiveness of the call-recall cytology-based programs. ${ }^{63}$ The equality of self-collected and clinician-collected HPV samples has been demonstrated by a recent meta-analysis, which found that the two collection methods had similar sensitivity and specificity for the detection of CIN2+ when polymerase chain reaction-based tests are used. ${ }^{64}$ Furthermore, the results from a recent study conducted in South Africa support the equality of the two methods among HIV-infected women. ${ }^{65}$

By overcoming some of the main institutional barriers to $\mathrm{CC}$ screening, such as limited access to health services,

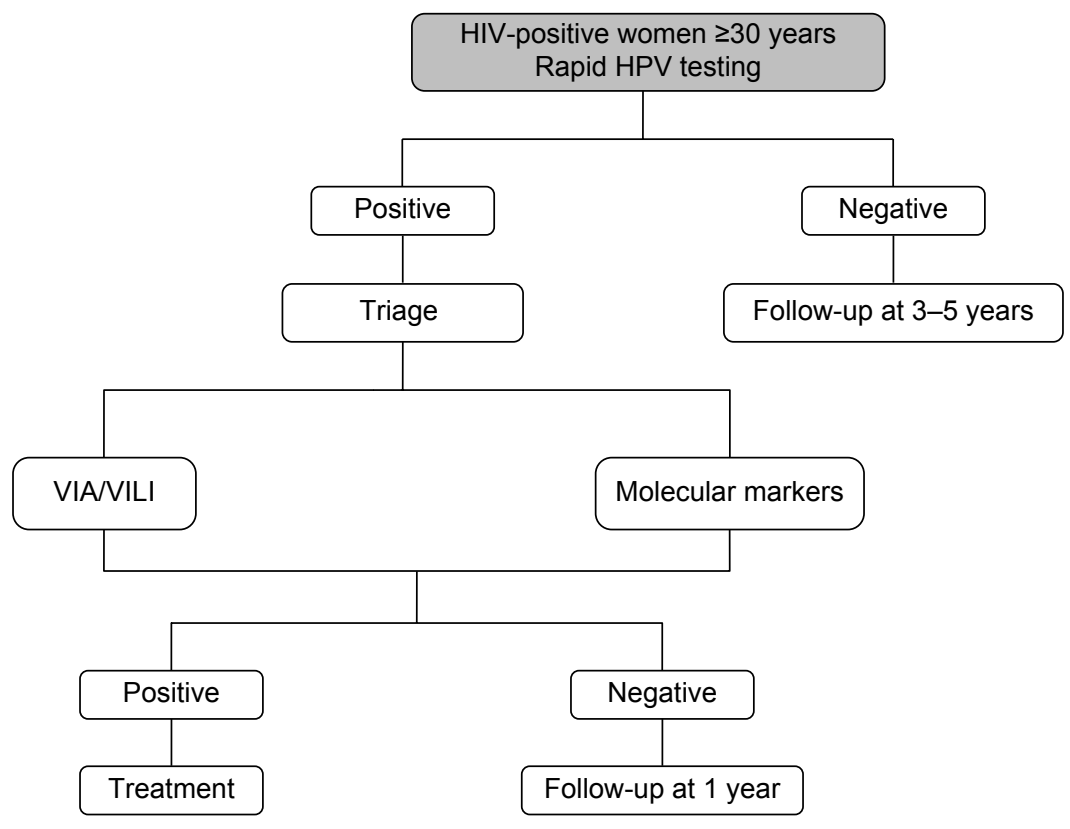

Figure 4 Decision-making algorithm for HPV-based screening.

Abbreviations: HIV, human immunodeficiency virus; HPV, human papillomavirus; VIA, visual inspection with acetic acid; VILI, visual inspection with Lugol's iodine. 
Table 3 Advantages and disadvantages of different CC screening options

\begin{tabular}{lll}
\hline Variable & Cytology & VIA HPV testing \\
\hline Advantages & & \\
High specificity & & \\
Feasible integration into a same day & & \\
screen-and-treat approach & & \\
Avoids the need of a pelvic & & \\
examination & & \\
High sensitivity & & \\
Disadvantages & & \\
Screening requires more than & $X$ \\
one visit & & \\
Need important health care & $X$ & \\
infrastructure & & \\
Need important laboratory supplies & $X$ & \\
Need qualified personnel for results & $X$ & \\
interpretation & & \\
High FN rates resulting in the need & $X$ & \\
to repeat the test \\
High FP rates resulting in a higher \\
risk of overtreatment
\end{tabular}

Abbreviations: CC, cervical cancer; FN, false negative; FP, false positive; HIV, human immunodeficiency virus; HPV, human papillomavirus; VIA, visual inspection with acetic acid.

the time-consuming aspect of a multiple-visit approach and the discomfort that can derive from a pelvic examination, the HPV self-sampling approach represents a mean to potentially increase CC screening participation. A recent randomized trial conducted in the USA has demonstrated that offering HPV self-sampling and subsequent results counseling within the same day increases the HIV-positive women's perception of their CC risk. ${ }^{66}$ Another study conducted in Uganda has found that self-sampling was well accepted by HIV-infected women, achieving screening attendance rates of $>90 \%{ }^{67}$ The overall advantages and disadvantages associated to the different screening techniques are reported in Table 3.

\section{Screening uptake}

Compared to industrialized countries, where CC screening covers $>60 \%$ of the target population, screening in developing countries covers $<20 \%$ of women. ${ }^{68}$ A recent review has estimated that the screening coverage rates in sub-Saharan Africa range from $2 \%$ to $20.2 \%$ in urban areas and from $0.4 \%$ to $14 \%$ in rural areas. ${ }^{69}$ More specifically South Africa, which accounts for the highest burden of HIV in the world, has a CC screening uptake that was estimated to be 54\% in 2013, with local variations that went from a minimum of $32 \%$ to a maximum of $75 \% .65,70$

The main intrapersonal obstacles that keep HIV-positive women from undergoing screening include an expected feeling of pain, discomfort, and anxiety that derive from the initial pelvic examination and the subsequent colposcopy in case of a positive screening test. ${ }^{71}$ In addition, older age and a perceived low risk of developing $\mathrm{CC}$ have also proven to be obstacles to screening participation. ${ }^{68,72}$ Institutional barriers, defined by a limited access to transportation and health care facilities, an extensive waiting time, and systemic issues related to scheduling multiple gynecological appointments, also stand in the way of women attending CC screening. ${ }^{73}$ There are also socioeconomic factors, such as the cost of the screening service and of the transportation to the health facilities, which may be necessary multiple times depending on the primary test results and the type of strategy that is adopted that can have a negative impact on screening participation. ${ }^{68}$

Most of the aforementioned institutional and intrapersonal barriers to adequate screening attendance apply to HIV-infected women as much as to their HIV-negative counterparts. The main advantage in favor of HIV-infected women is the fact that their more frequent health facility attendance can be viewed as an opportunity to provide health education, screening, and treatment of cervical precancerous and cancerous lesions within the same facility and, possibly, the same day. According to health care providers, the integration of CC screening in this context would allow to break down the main intrapersonal and institutional barriers that withhold this high-risk group of women from undergoing screening. ${ }^{74}$

Several studies have proven that information is a key component to increasing screening uptake among women in LMIC. ${ }^{71,74,75}$ While a study conducted in Ethiopia has found that women are 4 times more likely to undergo $\mathrm{CC}$ screening if they have previously been diagnosed with a sexually transmitted disease, another study in Uganda has shown that having multiple medical appointments for HIV and screening-related services can generate a considerable amount of stress and confusion, thus discouraging screening participation. ${ }^{71,72}$ In addition, not knowing how a screening procedure is performed is responsible for speculations and misconceptions on the $\mathrm{CC}$ prevention pathway. ${ }^{71}$

A recent study conducted on HIV-infected women has shown that, despite the increase in screening participation up to $95 \%$ with the use of HPV self-sampling, women were more likely to be lost at the subsequent follow-up visits if they had severe immunosuppression, higher viral loads, and a history of substance abuse. ${ }^{66}$ A solid health education delivered through HIV services represents the fundamental basis for increasing self-awareness and, subsequently, screening attendance. One study conducted in Western Kenya has found that, 
while self-reported HIV was associated to increased CC screening attendance, not all women who acknowledged living with HIV underwent screening, thus highlighting the importance of education in this high-risk group of women. ${ }^{76}$ Growing evidence shows the importance of informing not only the women, but also their male partners, as their support can significantly influence the women's decision to undergo screening. ${ }^{74,75}$ One way to ensure the correct transmission of information and to retain patients in health care is by building strong provider-patient relationships, especially for patients with multiple medical and social needs. ${ }^{73}$

By integrating HIV health care and CC screening services, another one of the main obstacles that can be overcome is the women's reluctance to attend screening due to the distance to the health care facilities. One study has shown that women having received a pelvic examination for $\mathrm{CC}$ screening at a place other than their usual health facility for HIV care were more likely to not undergo screening in the future. ${ }^{77}$ With the progressive implementation of screen-and-treat strategies, this systemic issue is further tackled by providing HIV care, CC screening, and treatment possibly all within the same day. ${ }^{71}$

\section{Screening timing, intervals, and follow-up}

For HIV-infected women living in western countries, the $\mathrm{CC}$ screening guidelines normally referred to are those for the treatment and prevention of opportunistic infections in HIV-infected adults and adolescents endorsed by the CDC, the National Institutes of Health (NIH), and HIV Medicine Association (HIVMA) of the Infectious Diseases Society of America. The common denominator to these guidelines is the prevention of $\mathrm{CC}$ by screening, management of women with positive test results, and subsequent treatment of histologically confirmed high-grade cancer precursors. According to these recommendations, HIV-infected women should undergo 2 Pap smears within the first year after diagnosis of HIV infection, followed by annual Pap testing regardless of their age. ${ }^{78,79}$ As opposed to HIV-uninfected women, whose HPV prevalence progressively decreases with age, HIVpositive women have an HPV prevalence that remains high across different age groups, which justifies the decision to not yet define the age at which to interrupt screening for these women. ${ }^{80}$ Differently from their HIV-negative counterpart, HIV-positive women should undergo screening within 1 year of the onset of sexual activity even if $<21$ years of age. ${ }^{81}$

Similar to the guidelines that apply to high-income countries, the WHO recommendations also advise to start screening for $\mathrm{CC}$ as soon as the onset of sexual activity for women living in areas where the HIV prevalence is high and whose HIV status is either positive or unknown. ${ }^{82}$ Although the recent implementation of HPV testing justifies the prolongation of the screening interval from 3 to 5 years for screen-negative women, such consideration only applies to HIV-negative women. ${ }^{57,83}$ Despite the lack of evidence coming from LMIC, the higher risk of developing cervical precancerous and cancerous lesions for HIV-infected women justifies a closer follow-up schedule. The WHO currently suggests repeating screening after 3 years in presence of a negative screening test for this high-risk group of women. ${ }^{82}$

Although evidence on the subject is currently limited, the recurrence rates of CIN among HIV-infected women following standard excisional treatment have been proven to range from $20 \%$ to $75 \% .{ }^{84}$ The results of a retrospective cohort study have shown a recurrence rate of $18 \%$ among HIV-positive women treated with thermocoagulation and followed for a 4-year period. This result was not significantly different from that obtained for HIV-negative women in the same cohort. ${ }^{85}$

Despite the literature gap on the subject, it seems evident that a closer follow-up schedule is advised for women who either test positive at primary screening or who are treated for a cervical premalignant lesions. What current knowledge seems to suggest, however, is that the timing of such follow-up visits should be no different than that applied to HIV-negative women.

\section{Conclusion}

To date, the best CC screening approach for HIV-infected women living in sub-Saharan Africa, where the HIV epidemic reaches its peak, has yet to be defined. As the risks-benefits balance does not seem to weight more toward a certain screening strategy for HIV-infected women, current evidence suggests that the screening options applied to HIV-negative women should be extended to their HIV-positive counterparts. The higher risk of developing cervical precancerous and cancerous lesions, however, imposes a closer follow-up schedule for this group of women. ${ }^{82}$ The lack of sufficient evidence on the subject limits the reliability of the current $\mathrm{CC}$ screening guidelines for $\mathrm{HIV}$-infected women and, thus, imposes the need to conduct further studies in order to provide more solid foundations for the prevention of a disease that currently represents a public health issue.

\section{Disclosure}

The authors report no conflicts of interest in this work. 


\section{References}

1. Ngono G, Evina F. Enquête Démographique et de Santé et Indicateurs Multiples du Cameroun. Claverton, Maryland, USA: INS \& ICF International; 2011.

2. Allemani C, Weir HK, Harewood R, et al. Global surveillance of cancer survival 1995-2009: analysis of individual data for 25,676,887 patients from 279 population-based registries in 67 countries (CONCORD-2). Lancet. 2015;385(9972):977-1010.

3. Catarino R, Vassilakos P, Tebeu PM, Schafer S, Bongoe A, Petignat P. Risk factors associated with human papillomavirus prevalence and cervical neoplasia among Cameroonian women. Cancer Epidemiol. 2016;40:60-66.

4. data.worldbank.org. [homepage on the Internet]. The World Bank Group; 2016. Available from: http://data.worldbank.org/indicator/ SH.DYN.AIDS.ZS. Accessed September 29, 2016.

5. Mbulaiteye SM, Bhatia K, Adebamowo C, Sasco AJ. HIV and cancer in Africa: mutual collaboration between HIV and cancer programs may provide timely research and public health data. Infect Agent Cancer. 2011;6(1):16-18.

6. Shibuya K, Mathers CD, Boschi-Pinto C, Lopez AD, Murray CJ. Global and regional estimates of cancer mortality and incidence by site: II. Results for the global burden of disease 2000. BMC Cancer. 2002;2:37.

7. Fitzmaurice C, Dicker D, Pain A, et al. The global burden of cancer 2013. JAMA Oncol. 2015;1(4):505-527.

8. Ferlay J, Soerjomataram I, Ervik M, et al. GLOBOCAN 2012 v1.1, Cancer Incidence and Mortality Worldwide: IARC CancerBase No. 11 [Internet]. Available from: http://globocan.iarc.fr. Accessed July 22, 2016.

9. Joint United Nations Programme on HIV/AIDS (UNAIDS). "The Gap Report 2014". UNAIDS, Geneva, Switzerland; 2014. Available from: http://www.unaids.org/sites/default/files/media_asset/UNAIDS_Gap_ report_en.pdf. Accessed August 3, 2016.

10. Crosbie EJ, Einstein MH, Franceschi S, Kitchener HC. Human papillomavirus and cervical cancer. Lancet. 2013;382(9895):889-899.

11. Stein L, Urban MI, O'Connell D, et al. The spectrum of human immunodeficiency virus-associated cancers in a South African black population: results from a case-control study. Int J Cancer. 2008;(122): 2260-2265.

12. Choudhury SA, Choudhury NA, Humphrey AD, et al. Higher prevalence of Human Papillomavirus-related cervical precancerous abnormalities in HIV-infected compared to HIV-uninfected women. J Natl Med Assoc. 2016;108(1):10-23.

13. Ngandwe C, Lowe JJ, Richards PJ, Hause L, Wood C, Angeletti PC. The distribution of sexually transmitted Human Papillomaviruses in HIV positive and negative patients in Zambia. BMC Infect Dis. 2007;7:77.

14. Clifford GM, Franceschi S, Keiser O, et al. Immunodeficiency and the risk of cervical intraepithelial neoplasia $2 / 3$ and cervical cancer: a nested case-control study in the Swiss HIV cohort study. Int J Cancer. 2016;138(7):1732-1740.

15. Bekolo CE, O’Bryan G, Tchago FE, Nangue C, Bekoule PS, Kollo B. Integrating cervical cancer screening with HIV care in Cameroon: comparative risk analysis of cervical disease in HIV-infected women recieving antiretroviral therapy to women in the general population. PLoS One. 2016;11(2):e0149152.

16. Bucciardini R, Fragola V, Abegaz T, et al. Retention in care of adult HIV patients initiating antiretroviral therapy in Tigray, Ethiopia: a prospective observational cohort study. PLoS One. 2015;10(9):e0136117.

17. Yaya I, Landoh DE, Saka B, et al. Predictors to adherence to antiretroviral therapy among people living with HIV and AIDS at the regional hospital of Sokode, Togo. BMC Public Health. 2014;14:1308.

18. Cobucci RN, Lima PH, de Souza PC, et al. Assessing the impact of HAART on the incidence of defining and non-defining AIDS cancers among patients with HIV/AIDS: a systematic review. $J$ Infect Public Health. 2014;8(1):1-10.

19. World Health Organisation. Guideline on When to Start Antiretroviral Therapy and On Pre-Exposure Prophylaxis for HIV. WHO Library Cataloguing-in-Publication Data. WHO Press, Geneva, Switzerland; 2015.
20. Bendavid E, Bhattacharya J. The President's Emergency plan for AIDS relief in Africa: an evaluation of outcomes. Ann Intern Med. 2009; 150(10):688-665.

21. World Health Organization. Comprehensive Cervical Cancer Control: A Guide to Essential Practice. 2nd ed. WHO Library Cataloguing-inPublication Data. WHO Press, Geneva, Switzerland. 2014.

22. Sinayobye JA, Sklar M, Hoover DR, et al. Prevalence and risk factors for high-risk human papillomavirus (hrHPV) infection among HIVinfected and uninfected Rwandan women: implications for hrHPV-based screening in Rwanda. Infect Agents Cancer. 2014;9:40.

23. Safaeian M, Kiddugavu M, Gravitt PE, et al. Prevalence and risk factors for carcinogenic human papillomavirus infections in rural Rakai, Uganda. Sex Transm Infect. 2008;84(4):306-311.

24. Moodley JR, Hoffman M, Carrara H, et al. HIV and pre-neoplastic and neoplastic lesions of the cervix in South Africa: a case-control study. BMC Cancer. 2006;6:135.

25. Woodman CB, Collins SI, Young LS. The natural history of cervical HPV infection: unresolved issues. Nat Rev Cancer. 2007;7(1):11-22.

26. Segondy M, Kelly H, Djima F, et al. Performance of careHPV for detecting high-grade cervical intraepithelial neoplasia among women living with HIV-1 in Burkina Faso and South Africa: HARP study. Br J Cancer. 2016;115(4):425-430.

27. Jemal A, Center MM, DeSanctis C, Ward EM. Global patterns of cancer incidence and mortality rates and trends. Cancer Epidemiol Biomarkers Prev. 2010;19(8):1893-1907.

28. Jemal A, Fedewa SA. Is the prevalence of ER-negative breast cancer in the US higher among Africa-born than US-born black women? Breast Cancer Res Treat. 2012;135(3):867-873.

29. Chung MH, McKenzie K, De Vuyst H, et al. Comparing papanicolau smear, visual inspection with acetic acid and human papillomavirus cervical cancer screening methods among HIV-positive women by immune status and antiretroviral therapy. AIDS. 2013;27(18): 2909-2919.

30. Maiman M, Tarricone N, Vieira J, Suarez J, Serur E, Boyce JG. Colposcopic evaluation of human immunodeficiency virus seropositive women. Obstet Gynecol. 1991;78(1):84-88.

31. Fink MJ, Frucher RG, Maiman M, et al. The adequacy of cytology and colposcopy in diagnosing cervical neoplasia in HIV-seropositive women. Gynecol Oncol. 1994;55(1):133-137.

32. Sankaranarayanan R, Budukh AM, Rajkumar R. Effective screening programmes for cervical cancer in low- and middle-income developing countries. Bull World Health Organ. 2001;79(10):954-962.

33. Huchko MJ, Sneden J, Leslie HH, et al. A comparison of two visual inspection methods for cervical cancer screening among HIV-infected women in Kenya. Bull World Health Organ. 2014;92(3):195-203.

34. Cronjé HS. Screening for cervical cancer in developing countries. Int J Gynecol Obstet. 2004;84(2):101-108.

35. Anderson J, Wysong M, Estep D, et al. Evaluation of cervical cancer screening programs in Côte d'Ivoire, Guyana and Tanzania: effect of HIV status. PLoS One. 2015;10(9):e0139242.

36. Huchko MJ, Sneden J, Sawaya G, et al. Accuracy of visual inspection with acetic acid to detect cervical cancer precursors among HIV-infected women in Kenya. Int J Cancer. 2015;136(2):392-398.

37. Shiferaw N, Salvador-Davila G, Kassahun K, et al. The single-visit approach as a cervical cancer prevention strategy among women with HIV in Ethiopia. Successes and lessons learned. Glob Health Sci Pract. 2016;4(1):87-98.

38. Martin CE, Tergas AI, Wysong M, Reinsel M, Estep D, Varallo J. Evaluation of a single-vsit approach to cervical cancer screening and treatment in Guyana: feasibility, effectiveness and lessons learned. J Obstet Gynaecol Res. 2014;40(6):1707-1716.

39. Odafe $\mathrm{S}$, Torpey $\mathrm{K}$, Khamofu $\mathrm{H}$, et al. Integrating cervical cancer screening with HIV care in a district hospital in Abuja, Nigeria. Niger Med J. 2013;54(3):176-184.

40. Audet CM, Silva Matos C, Blevins M, Cardoso A, Moon TD, Sidat M. Acceptability of cervical cancer screening in rural Mozambique. Health Educ Res. 2012;27(3):544-551. 
41. Mwanahamuntu MH, Sashasrabuddhe VV, Blevins M, et al. Utilisation of cervical cancer screening services and trends in screening positivity rates in a "screen-and-treat" program integrated with HIV/AIDS care in Zambia. PLoS One. 2013;8(9):e74607.

42. Sankaranarayan R, Nene BM, Dinshaw KA, et al. A cluster randomized controlled trial of visual, cytology and human papillomavirus screening for cancer of the cervix in rural India. Int J Cancer. 2005;116(4): 617-623.

43. Qiao L, Li B, Long M, Wang X, Wang A, Zhang G. Accuracy of visual inspection with acetic acid and with Lugol's iodine for cervical cancer screening. Meta-analysis. J Obstet Gynaecol Res. 2015;41(9): $1313-1325$.

44. DeGregorio GA, Bradford LS, Manga S, et al. Prevalence, predictors and same day treatment of positive VIA enhanced by digital cervicography and histopathology results in a cervical cancer prevention program. PLoS One. 2016;11(6): 0157319.

45. Firnhaber C, Mao L, Levin S, et al. Evaluation of a cervicography-based program to ensure quality of visual inspection of the cervix in HIVinfected women in Johannesburg, South Africa. J Low Gen Tract Dis. 2015;19(1):7-11.

46. Mwanahamuntu M, Sahasrabuddhe V, Kapambwe S, et al. Advancing cervical cancer prevention initiatives in resource-constrained settings: insights from the Cervical Cancer Prevention Program in Zambia. PLoS Med. 2011;8(5):e1001032.

47. Lince-Deroche N, Phiri J, Michelow P, Smith JS, Firnhaber C. Costs and cost effectiveness of three approaches for cervical cancer screening among HIV-positive women in Johannesburg, South Africa. PLoS One. 2015;10(11):e0141969.

48. Dolman L, Sauvaget C, Muwonge R, Sankaranarayanan R. Metaanalysis of the efficacy of cold coagulation as a treatment method for cervical intraepithelial neoplasia: a systematic review. BJOG. 2014; 121(8):929-924.

49. Sanjaranarayan R, Basu P, Wesley RS, et al. Accuracy of visual screening for cervical neoplasia: results from an IARC multicenter study in India and Africa. Int $J$ cancer. 2004;110(6):907-913.

50. Chibwesha CJ, Frett B, Katundu K, et al. Clinical performance validation of 4 point-of-care cervical cancer screening tests in HIV-infected women in Zambia. J Low Gen Tract Dis. 2016;20(3):218-223.

51. Denny L, Anorlu R. Cervical cancer in Africa. Cancer Epidemiol Biomarkers Prev. 2012;21(9):1434-1438.

52. Einstein MH, Smith KM, Davis TE, et al. Clinical evaluation of the cartridge-based GeneXpert human papillomavirus assay in women referred for colposcopy. J Clin Microbiol. 2014;52(6): 2089-2095.

53. McDonald A, Tergas AI, Kuhn L, Denny L, Wright TC Jr. Distribution of human papillomavirus genotypes among HIV-positive and HIV-negative women in Cape Town, South Africa. Front Oncol. 2014;4:48.

54. Giorgi-Rossi P, Franceschi S, Ronco G. HPV prevalence and accuracy of HPV testing to detect high-grade cervical intraepithelial neoplasia. Int J Cancer. 2012;130(6):1387-1394.

55. Franceschi S, Herrero R, CLifford GM, et al. Variations in the agespecific curves of human papillomavirus prevalence in women worldwide. Int J Cancer. 2006;119(11):2677-2684.

56. Schiffman M, Wentzensen N, Wacholder S, Kinney W, Gage JC, Castle PE. Human papillomavirus testing in the prevention of cervical cancer. J Natl Cancer Inst. 2011;103(5):368-383.

57. Saslow D, Solomon D, Lawson HW, et al. American Cancer Society, American Society for Colposcopy and Cervical Pathology, and American Society for Clinical Pathology screening guidelines for the prevention of early detection of cervical cancer. Am J Clin Pathol. 2012; 137(4):516-542.

58. Mujuni F, Mirambo MM, Rambau P, et al. Variability of high risk HPV genotypes among HIV infected women in Mwanza, Tanzania- the need for evaluation of current vaccine effectiveness in developing countries. Infect Agent Cancer. 2016;11:49.

59. Cattani P, Zannoni GF, Ricci C, et al. Clinical performance of human papillomavirus E6 and E7 mRNA testing for high-grade lesions of the cervix. J Clin Microbiol. 2009;47(12):3895-3901.
60. De Vuyst H, Franceschi S, Plummer M, et al. Methylation levels of CADM1, MAL, and MIR124-2 in cervical scrapes for triage of HIVinfected, high-risk HPV-positive women in Kenya. J Acquir Immune Defic Syndr. 2015;70(3):311-318.

61. Del Gobbo A, Bonoldi E, Cribiu' FM, et al. Insuline-like growth factor II mRNA binding protein 3 (IMP3) expression in cervical intraepithelial neoplasia and its relationship with HIV-infection status. Sex Health. 2015;12(1):22-26.

62. Gravitt PE, Rositch AF. HPV self-testing and cervical cancer screening coverage. Lancet Oncol. 2014;15(2):128-129.

63. Snijders PJ, Verhoef VM, Abryn M, et al. High-risk HPv testing on self-sampled versus clinician-collected specimens: a review on the clinical accuracy and impact on population attendance in cervical cancer screening. Int J Cancer. 2013;132(10):2223-2236.

64. Arbyn M, Verdoot F, Snijders PJ, et al. Accuracy of human papillomavirus testing on self-collected versus clinician-collected samples: a meta-analysis. Lancet Oncol. 2014;15(2):172-183.

65. Adamson PC, Huchko MJ, Moss AM, Kinkel HF, Medina-Marino A. Acceptability and accuracy of cervical cancer screening using a selfcollected tampon for HPV messenger-RNA testing among HIV-infected women in South Africa. PLoS One. 2015;10(9):e0137299.

66. Murphy J, Mark H, Anderson J, Farley J, Allen J. A randomized trial of human papillomavirus self-sampling as an intervention to promote cervical cancer screening among women with HIV.J Low Gen Tract Dis. 2016; 20(2):139-144.

67. Serwadda D, Wawer MJ, Shah KV, et al. Use of a Hybrid capture assay of self-collected vaginal swabs in rural Uganda for detection of human papillomavirus. J Infect Dis. 1999;180(4):1316-1319.

68. Gakidou E, Nordhagen S, Obermeyer Z. Coverage of cervical cancer screening in 57 countries: low average levels and large inequalities. PLoS Med. 2008;5(6):e132.

69. Louie KS, de Sanjose S, Mazaud P. Epidemiology and prevention of human papillomavirus and cervical cancer in sub-Saharan Africa: a comprehensive review. Trop Med Int Health. 2009;14(10): $1287-1302$

70. Duerr A, Kieke B, Warren D, et al. Human papillomavirus-associated cervical cytologic abnormalities among women with or at risk of infection with human immunodeficiency virus. Am J Obstet Gynecol. 2001; 184(4):584-590.

71. Bukirwa A, Mutyoba JN, Mukasa BN, et al. Motivations and barriers to cervical cancer screening among HIV infected women in HIV care: a qualitative study. BMC Womens Health. 2015;15:82.

72. Bayu H, Berhe Y, Mulat A, Alemu A. Cervical cancer screening service uptake and associated factors among age eligible women in Mekelle Zone, Northern Ethiopia, 2015: A community based study using health belief model. PLoS One. 2016;11(3):e0149908.

73. Fletcher FE, Buchberg M, Schover LR, et al. Perceptions of barriers and facilitators to cervical cancer screening among low-income, HIVinfected women from an integrated HIV clinic. AIDS Care. 2014;26(10): 1229-1235.

74. Kumakech E, Andersson S, Wabinga H, Berggren V. Integration of HIV and cervical cancer screening perceptions of healthcare providers and policy makers in Uganda. BMC Public Health. 2014; 14:810

75. Ogunwale AN, Coleman MA, Sangi-Haghpeykar H, et al. Assessment of factors impacting cervical cancer screening among low-income women living with HIV-AIDS. AIDS Care. 2016;28(4):491-494.

76. Orango'o EO, Wachira J, Asirwa FC, et al. Factors associated with uptake of visual inspection with acetic acid (VIA) for cervical cancer screening in Western Kenya. PLoS One. 2016;11(6):e0157217.

77. Frazier EL, Sutton MY, Tie Y, McNaghten AD, Blair JM, Skarbinski J. Screening for cervical cancer and sexually transmitted diseases among HIV-infected women. $J$ Womens Health (Larchmt). 2016;25(2): 124-132.

78. Moscicki AB, Cox JT. Practice improvement in cervical screening and management (PICSM): symposium on management of cervical abnormalities in adolescents and young women. J Low Genit Tract Dis. 2010;14(1):73-80. 
79. AIDSinfo. Guidelines for Prevention and treatment of opportunisitc infections in HIV-infected adults and adolescents: recommendations from CDC, the National Institutes of Health, and the HIV medicine association of the infectious diseases society of America. MMWR. 2009; 58(RR-4):1-207.

80. Mbulawa ZZA, Coetzee D, Williamson AL. Human papillomavirus prevalence in South African women and men according to age and human immunodeficiency virus status. BMC Infect Dis. 2015;15:459.

81. Keller MJ. Screening for human papillomavirus-associated cervical disease in HIV-infected women. Top Antivir Med. 2015;23(4): 142-145.

82. Santesso N, Mustafa RA, Schünemann HJ, et al. World Health Organization guidelines for treatment of cervical intraepithelial neoplasia 2-3 and screen-and-treat stretegies to prevent cervical cancer. Int J Gyn Obstet. 2016;132(3):252-258.

83. Adler DH, Wallace M, Bennie T, et al. Cervical dysplasia and high-risk human papillomavirus infections among HIV-infected and HIV-uninfected adolescent females in South Africa. Infect Dis Obstet Gynecol. 2014;2014:498048.

84. Tebeu PM, Major AL, Mhawech P, Rapiti E. The recurrence of cervical intraepithelial neoplasia in HIV-positive women: a review of the literature. Int J STD AIDS. 2006;17(8):507-511.

85. Oga EA, Brown JP, Brown C, et al. Recurrence of cervical intraepithelial lesions after thermo-coagulation in HIV-positive and HIV-negative Nigerian women. BMC Womens Health. 2016;16:25.

86. Hotez PJ, Fenwick A, Kjetland EF. Africa's 32 cents solution for HIVAIDS. PLoS Negl Trop Dis. 2009;3(5):e430.
87. Ferlay J, Shin HR, Bray F, Forman D, Mathers C, Parkin DM. GLOBOCAN 2008, Cancer Incidence and Mortality Worldwide: IARC CancerBase No. 10 [Internet]. Lyon, France: International Agency for Research on Cancer; 2010. Available from: https://www.iarc.fr/en/media-centre/ iarcnews/2010/globocan2008.php. Accessed January 13, 2017.

88. Akinwuntwan L, Adesina OA, Okolo CA, et al. Correlation of cervical cytology and visual inspection with acetic acid in HIV-positive women. J Obstet and Gynaecol. 2008;28(6):638-641.

89. Mabeya H, Khozaim K, Liu T, et al. Comparison of conventional cervical cytology versus visual inspection with acetic acid (VIA) amon HIV-infected women in western Kenya. J Low Gen Tract Dis. 2012;16(2):92-97.

90. Kuhn L, Wang C, Tsai WY, Wright TC, Denny L. Efficacy of human papillomavirus-based screen-and-treat for cervical cancer prevention among HIV-infected women. AIDS. 2010;24(16):2553-2561.

91. Firnhaber C, Mayisela N, Mao L, et al. Validation of cervical cancer screening methods in HIV positive women from Johannesburg South Africa. PLoS One. 2013;8(1):e53494.

92. Dartell MA, Rasch V, Ifner T, et al. Performance of visual inspection with acetic acid and human papillomavirus testing for detection of high-grade cervical lesions in HIV positive and HIV negative Tanzanian women. Int J Cancer. 2014;135(4):896-904.

93. Ngou J, GIlham C, Omar T, et al. Comparison of analytical and clinical performances of the digene HC2 HPV DNA assay and the INNO-LiPA HPV genotyping assay for detecting high-risk HPV infection and cervical neoplasia among HIV-positive African women. J Acquir Immune Defic Syndr. 2010;68(2):162-168.
International Journal of Women's Health

\section{Publish your work in this journal}

The International Journal of Women's Health is an international, peerreviewed open-access journal publishing original research, reports, editorials, reviews and commentaries on all aspects of women's healthcare including gynecology, obstetrics, and breast cancer. The manuscript management system is completely online and includes

\section{Dovepress}

a very quick and fair peer-review system, which is all easy to use. Visit http://www.dovepress.com/testimonials.php to read real quotes from published authors. 